\title{
An Unusual Cause of Refractory Hypoxemia in Cirrhosis
}

\author{
Yashwant Patidar MD and Sachin Kumar MD DM
}

\begin{abstract}
Pulmonary gas exchange abnormalities and refractory hypoxemia cause myriad difficulties in patients with chronic liver disease. In addition to intrinsic cardiopulmonary diseases and hepatopulmonary syndrome, some unusual pathophysiological mechanisms in patients with portosystemic collaterals might contribute to hypoxemia. We report the clinical presentation of an unusual portosystemic anatomic shunt that permits venous admixture with oxygenated blood, causing hypoxemia that is refractory to the administration of supplemental oxygen, and recurrent hepatic encephalopathy. There has been no such report in the published literature. This case highlights the importance of keeping direct portopulmonary venous anastomosis in the differential diagnosis of oxygen-refractory hypoxemia and recurrent hepatic encephalopathy in patients with cirrhosis in the appropriate clinical context. Key words: hypertension; portal; hypoxemia; hepatic encephalopathy; contrast echocardiography; portosystemic shunt; cirrhosis. [Respir Care 2015;60(3):e49-e51. () 2015 Daedalus Enterprises]
\end{abstract}

\section{Introduction}

Pulmonary gas exchange abnormalities and hypoxemia are common in patients with chronic liver disease. In addition to intrinsic cardiopulmonary disorders, a variety of causes of hypoxemia in liver disease have been identified. These include unique problems associated with the presence of liver disease and/or portal hypertension, such as intrapulmonary right-to-left shunt in hepatopulmonary syndrome and intracardiac shunt with predominately right-toleft flow, which may accompany atrial or ventricular septal defects with Eisenmenger physiology. ${ }^{1}$ In this report, we describe an unusual cause of unexplained hypoxia and hyperammonemia leading to recurrent hepatic encephalopathy.

\footnotetext{
Dr Patidar is affiliated with the Department of Radiodiagnosis, and Dr Kumar is affiliated with the Department of Pulmonary Medicine, Institute of Liver and Biliary Sciences, New Delhi, India.
}

The authors have disclosed no conflicts of interest.

Correspondence: Sachin Kumar MD DM, Department of Pulmonary Medicine, Institute of Liver and Biliary Sciences, Sector D-1, Vasant Kunj, New Delhi 110070, India. E-mail: sachin.drk@gmail.com, drsachin.kumar@ilbs.in.

DOI: $10.4187 /$ respcare.03529

\section{Case Report}

A 43-y-old male with hepatitis $\mathrm{C}$ virus-related cirrhosis and recurrent hepatic encephalopathy was referred for evaluation of dyspnea. Physical examination determined grade 3 clubbing and oxygen saturation of $87 \%$. His chest $\mathrm{x}$-ray and echocardiogram were normal. Spirometry revealed normal percent-of-predicted values for $\mathrm{FEV}_{1}(86 \%)$, FVC (94\%), and $\mathrm{FEV}_{1} / \mathrm{FVC}(0.92)$, with a moderately reduced diffusing capacity for carbon monoxide $(10.8 \mathrm{~mL} / \mathrm{min} / \mathrm{mm} \mathrm{Hg}, 44 \%$ of predicted). His plasma ammonia level was $294 \mu \mathrm{g} / \mathrm{dL}$ (normal values, $12-60 \mu \mathrm{g} / \mathrm{dL}$ ). Arterial blood gas analysis revealed a $\mathrm{pH}$ of 7.42 , a $\mathrm{P}_{\mathrm{CO}_{2}}$ of $28 \mathrm{~mm} \mathrm{Hg}$, and a $\mathrm{P}_{\mathrm{O}_{2}}$ of $52 \mathrm{~mm} \mathrm{Hg}$ with an alveolar-arterial difference of $58 \mathrm{~mm} \mathrm{Hg}$ (on room air). His oxygen saturation did not improve on supplemental oxygen (initially $3 \mathrm{~L} / \mathrm{min}$ supplied via nasal cannula and later with non-rebreathing mask). Saline contrast echocardiography was negative.

During further evaluation, the patient underwent triplephase computed tomography of the chest and abdomen, which showed a direct shunt connecting the right portal vein to the left atrium via the right inferior pulmonary vein, resulting in a right-to-left extracardiac shunt (Fig. 1). The patient was managed conservatively and discharged later.

\section{Discussion}

Portal hypertension is a progressive inevitable consequence of cirrhosis with formation of many collaterals. ${ }^{2}$ 


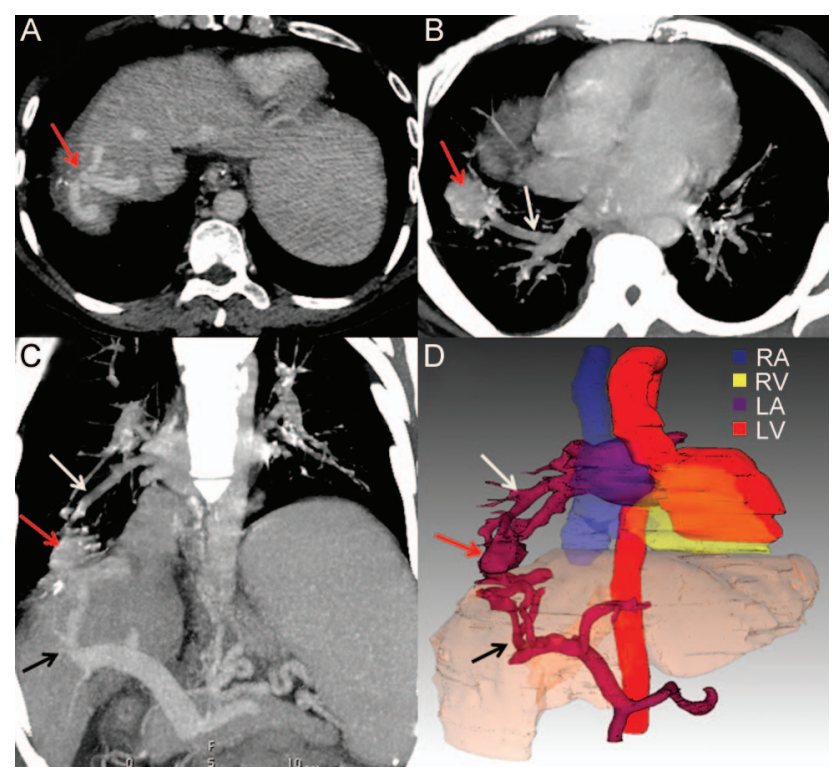

Fig. 1. Axial (A and B), oblique coronal (C), and 3-dimensional maximum intensity projection (MIP) with volume-rendered (D) contrast-enhanced computed tomography images of the chest and upper abdomen revealed a cluster of collaterals (red arrows) from the anterior and posterior branches of the right portal vein (black arrows in $\mathrm{C}$ and $\mathrm{D}$ ) involving the posterior segment of the right lobe of the liver and extending through the diaphragm up to the anterior-basal segment of the right lower lobe and ultimately draining into the left atrium via the right inferior pulmonary vein (white arrows in B-D). See D for cardiac chamber key.

Portopulmonary collaterals, also called portopulmonary venous anastomoses, connect with the pulmonary circulation. We highlighted the clinical presentation of an unusual anatomic shunt that permits venous admixture with oxygenated blood, causing hypoxemia that is refractory to the administration of supplemental oxygen, and recurrent hepatic encephalopathy.

In portal hypertension, collaterals can be either intrahepatic or extrahepatic. Dynamic contrast computed tomography is regarded as the best tool for evaluation of the status of portosystemic collaterals in patients with portal hypertension. ${ }^{3,4}$ Portopulmonary venous anastomosis has been recognized as one of the cephalad hepatofugal collaterals in patients with portal hypertension. ${ }^{5}$ Portopulmonary venous anastomosis was first reported by Schoenmackers and Vieten in 1953. ${ }^{6}$ Incidences have been reported to be $1-30 \%$ in portal hypertension..$^{5,7,8}$ In previous reports, portopulmonary venous anastomosis was detected on postmortem angiography and percutaneous transhepatic portography. However, computed tomography imaging of direct portopulmonary shunts has not been described in the literature.

Saline contrast echocardiography is used widely in screening intracardiac and intrapulmonary shunts. However, portopulmonary venous anastomosis is typically de- tected during transhepatic portography rather than saline contrast echocardiography because it provides enough contrast echoes to be picked up even at a location distant from the injection site. ${ }^{5}$ This explains the negative contrast echocardiography study in our case.

Extrapulmonary accumulation of Technetium-99m macroaggregated albumin (MAA) is also used to detect rightto-left shunts. The appearance of the radiotracer in the systemic circulation to document visualization of brain, kidneys, and spleen after intravenous administration of MAA indicates right-to-left intrapulmonary or intracardiac shunts because macroaggregated albumin particles $(20-$ $60 \mu \mathrm{m})$ supposedly bypass the pulmonary bed $(<15 \mu \mathrm{m})$ in these cases, instead of becoming trapped in a normal scenario without such shunts. ${ }^{9}$ However, the MAA scan was also expected to be negative in our case, as there was no intrapulmonary shunting, and macroaggregated albumin particles would still be trapped in the normal pulmonary bed.

When performing contrast echocardiography by the portal approach, 3 main routes might produce contrast echoes in the left heart: (1) portopulmonary venous anastomosis, (2) intracardiac right-to-left shunt, and (3) intrapulmonary right-to-left shunt (hepatopulmonary syndrome). The latter 2 produce contrast echoes first in the right cardiac chambers via the portacaval route and subsequently in the left cardiac chambers. Intracardiac shunt requires one cardiac beat or less, whereas intrapulmonary shunt requires a time interval of 4-6 cardiac beats. ${ }^{10,11}$ Portopulmonary venous anastomoses show characteristic contrast echo patterns: an earlier and stronger appearance of echoes in the left cardiac chambers than in the right. These results allow realtime differential diagnosis of portopulmonary venous anastomosis from other causes of intrapulmonary shunt. ${ }^{5}$

As the portal vein carries deoxygenated blood, portopulmonary venous anastomosis causes venous admixture, resulting in a significant reduction in arterial oxygen saturation, especially when the portal venous blood has very low oxygen saturation and/or the shunt is large enough to carry a significant amount of cardiac output. ${ }^{8,12}$ The hemodynamics in such cases involves a functional right-toleft shunt. Embolic and infective agents that migrate to the draining vein from the portal circulation have the potential to directly reach the left atrium through the [redundant] portopulmonary venous anastomosis, and systemic embolization can occur. Patients with portosystemic shunts may in fact present with neurological abnormalities and high ammonia levels even in the absence of significant liver damage. ${ }^{13}$ The passage of neurotoxic substances coming from the bowel into the systemic circulation can be involved in the pathogenesis of recurrent hepatic encephalopathy, as in our case. ${ }^{14}$

We reported the first case of direct spontaneous portopulmonary venous anastomosis connecting the portal vein 


\section{Hypoxemia in Chronic Liver Disease}

to the pulmonary veins and subsequently draining into the left atrium. This case highlights the importance of considering direct portopulmonary venous anastomosis in the differential diagnosis of oxygen-refractory hypoxemia and recurrent hepatic encephalopathy in patients with cirrhosis and the use of contrast-enhanced computed tomography in the ultimate diagnosis.

\section{REFERENCES}

1. Stoller JK, Hoffman RM, White RD, Mee RB. Anomalous hepatic venous drainage into the left atrium: an unusual cause of hypoxemia. Respir Care 2003;48(1):58-62.

2. Bolondi L, Gaiani S, Gebel M. Portohepatic vascular pathology and liver disease: diagnosis and monitoring. Eur J Ultrasound 1998;7 Suppl 3:S41-S52.

3. Atasoy C, Akyar S. Multidetector CT: contributions in liver imaging. Eur J Radiol 2004;52(1):2-17.

4. Ko JM, Ahn MI, Han DH, Jung JI, Park SH. Dynamic CT and MRA findings of a case of portopulmonary venous anastomosis (PPVA) in a patient with portal hypertension: a case report and review of the literature. Acta Radiol 2011;52(5):566-569.

5. Sano A, Nishizawa S, Sasai K, Imanaka K, Tanaka K, Hashimura T, et al. Contrast echocardiography in detection of portopulmonary venous anastomosis. AJR Am J Roentgenol 1984;142(1):137-140.

6. Schoenmackers J, Vieten H. [Porto-caval and porto-pulmonary anastomoses in postmortem portography]. Fortschr Geb Rontgenstr 1953; 79(4):488-498. Article in German.
7. Sano A, Kuroda Y, Moriyasu F, Takahashi Y, Koizumi S, Kimura S, Okuda K. Porto-pulmonary venous anastomosis in portal hypertension demonstrated by percutaneous transhepatic cine-portography. Radiology 1982;144(3):479-484.

8. Calabresi P, Abelmann WH. Porto-caval and porto-pulmonary anastomoses in Laennec's cirrhosis and in heart failure. J Clin Invest 1957;36(8):1257-1265

9. Lu G, Shih WJ, Chou C, Xu JY. Tc-99m MAA total-body imaging to detect intrapulmonary right-to-left shunts and to evaluate the therapeutic effect in pulmonary arteriovenous shunts. Clin Nucl Med 1996;21(3):197-202.

10. Attaran RR, Ata I, Kudithipudi V, Foster L, Sorrell VL. Protocol for optimal detection and exclusion of a patent foramen ovale using transthoracic echocardiography with agitated saline microbubbles. Echocardiography 2006;23(7):616-622.

11. Rollán MJ, Muñoz AC, Pérez T, Bratos JL. Value of contrast echocardiography for the diagnosis of hepatopulmonary syndrome. Eur J Echocardiogr 2007;8(5):408-410.

12. Kumar A, Gonzalez G, Wilkinson L, Mohammed TL, Castro-Pavia F, Glockner J, Kirsch J. Computed tomography findings of spontaneous porto-pulmonary shunts in 3 patients with portal hypertension. J Thorac Imaging 2010;25(3):W70-W74.

13. Crespin J, Nemcek A, Rehkemper G, Blei AT. Intrahepatic portalhepatic venous anastomosis: a portal-systemic shunt with neurological repercussions. Am J Gastroenterol 2000;95(6):1568-1571.

14. Riggio O, Efrati C, Catalano C, Pediconi F, Mecarelli O, Accornero $\mathrm{N}$, et al. High prevalence of spontaneous portal-systemic shunts in persistent hepatic encephalopathy: a case-control study. Hepatology 2005;42(5):1158-1165. 